\title{
Achievements in Slow and Fast Light in Optical Fibres
}

\author{
Luc Thévenaz \\ Ecole Polytechnique Fédérale de Lausanne (EPFL), Institute of Electrical Engineering \\ STI-GR-SCI Station 11, 1015 Lausanne, Switzerland \\ Tel: +41 21693 4774,Fax: +41 21693 2614,e-mail:Luc.Thevenaz@EPFL.CH
}

\begin{abstract}
The ubiquitous role occupied by optical fibres in modern photonic systems has steadily stimulated research to realize slow and fast light devices directly in this close-to-perfect transmission line. This potentially offers the key advantage of a direct and flexible integration in most existing optical systems. The recent progress in the realization of optically-controlled delays in optical fibres, under normal environmental conditions and in the high-transparency spectral region, has paved the way towards real applications based on slow and fast light. This presentation shows the state-of-the-art research in this fascinating field and the possible outcomes in the near future.
\end{abstract}

Keywords: fiber optics, nonlinear optics, slow and fast light, stimulated Brillouin scattering.

\section{INTRODUCTION AND PRINCIPLES}

Silica optical fibres have outperformed all existing transmission lines and have decisively contributed to the tremendous expansion of the global communication galaxy. The success is certainly due to their unrivalled performance in terms of their low optical loss, their wide bandwidth that allows terabits per second of information transmitted over more than $1000 \mathrm{~km}$, and also their extremely low production cost. Realizing tuneable delays directly in optical fibres and plugging this timing device readily in existing networks or systems is certainly a very attractive approach for the timing of data transmission, as well as for plain optical and microwave-on-optical-carrier signal processing. Considering the very classical type of propagation in standard single-mode fibres and the limited possibility to engineer the dispersion curve without creating complex photonic crystal structures, the research has been naturally directed towards solutions based on spectral resonances to generate slow and fast light.

The pioneering demonstrations of slow and fast light were all based on the exploitation of narrow spectral resonances, mainly created by electromagnetically-induced transparency[1] or coherent population oscillation[2]. Narrow spectral resonances induce an anomalous situation for the optical propagation, since any sharp spectral change in the medium transmission results in a steep linear variation of the effective refractive index along wavelength. This in turn results in a strong group velocity change at the exact centre of the resonance[3]. The effect of slow and fast light turns out to be the strongest for narrower spectral resonances, as explained here after. For this basic reason, the early experiments were all carried out in special media, such as ultra-cold atomic gases or atomic transitions in a crystalline solid working at a well defined wavelength.

Most spectral resonances can be described by a Lorentzian distribution as a function of the optical frequency $v$ and in this important case the relationship between the change in the optical transmission and the delay can be explicitly established. The refractive index change $\Delta n$ associated to the sharp spectral change in the medium transmission is conveniently calculated using the Kramers-Kronig transformation and the group index change $\Delta n_{g}$ is then immediately found using the conventional relationship $\Delta n_{g}=\Delta n+v(d \Delta n / d v)$ [3]. Finally, the delay $\Delta T$ added to the normal transit time in the medium is straightforwardly deduced from the group velocity change:

$$
\Delta T=\Delta n_{g} \frac{L}{c}=\frac{G}{2 \pi \Delta v} \quad \text { with } \quad G=\gamma L
$$

where $L$ is the propagation distance in the medium in metre, $\Delta v$ is the half width at half maximum of the Lorentzian distribution in $\mathrm{Hz}$ and $\gamma$ is the peak value of the optical transmission at the resonance centre in $\mathrm{m}^{-1}$. It must be pointed out that $\gamma$ is positive in a gain situation and so is the delay $\Delta T$ implying the existence of signal retardation and the creation of slow light, whilst in the case of absorption, $\gamma$ and $\Delta T$ are negative and signal advancement is observed under fast light conditions. Here, $G$ represents the total linear transmission - positive in the case of optical amplification and negative for absorption - and the signal power is thus changed by a factor of $e^{G}$ after propagation through the medium. This shows that delay and amplitude changes are tightly related when using a single spectral resonance indicating that delaying with a flat amplitude response will require more sophisticated schemes. Equation (1) also shows that the delaying strength is dependent on the linewidth, $\Delta v$, of the resonance and the total linear transmission, $G$, and that the strongest slow and fast light effect can be obtained in the centre of narrow and strong spectral lines.

Delay, however, cannot be extended arbitrarily since there is always a trade-off of strong dispersion associated to the presence of the spectral resonance. In most situations this dispersion manifests as pulse 
broadening contained in a data flow and sets a limit to the maximal delay with a tolerable broadening. In practice the maximal delay can only amount to one to two pulse widths and breaking this deadlock remains a major challenge in slow light based on spectral resonances.

The main obstacle for the creation of narrow spectral resonances in optical fibres is related to the highly disordered nature of the amorphous silica constituting the fibre material, prohibiting the use of narrow-band atomic transitions without a proper doping. The most efficient approach to create a narrow-band gain or loss in optical fibres remains the exploitation of an optical interaction that requires a severe phase matching condition to be satisfied. This way the waves interact only over the very limited spectral range where their amplitudes couple mutually and constructively. This can be realized using the nonlinear optical response of the material that offers the possibility to transfer the energy from one optical wave to another optical wave. For instance, a resonant coupling over a narrow frequency range is observed in parametric interactions, which are always conditioned to the generation of a third idler wave to satisfy the phase matching condition. The optical wave that benefits from the energy transfer will actually experience a linear gain and be subject to light slowing while the depleted wave will undergo a linear loss and will experience a fast light effect[3].

\section{SLOW \& FAST LIGHT IN OPTICAL FIBRES USING STIMULATED BRILLOUIN SCATTERING}

Among all parametric processes observed in silica, stimulated Brillouin scattering (SBS) turns out to be the most efficient. In its most simple configuration the coupling is realized between two optical waves propagating exclusively in opposite directions in a single mode fibre, through the stimulation by electrostriction of a longitudinal acoustic wave that plays the role of the idler wave in the interaction [4]. This stimulation is efficient only if the two optical waves show a frequency difference giving a beating interference resonant with an acoustic wave (that is actually never directly observed). This acoustic wave in turn induces a dynamic Bragg grating in the fibre core that diffracts the light from the higher frequency wave back into the wave showing the lower frequency.

The first experimental demonstrations of slow and fast light in optical fibres were achieved using SBS[5] first AT EPFL in Switzerland[6] and independently a few months later at Cornell University in USA [7]. This approach demonstrated that efficient delays can be realized using SBS. It also turned out to be an excellent platform to test the validity of the theoretical models describing slow and fast light, regarding the perfect Lorentzian distribution of the spectral resonance and the well controlled experimental conditions that can be implemented. For instance, the predicted delay of $1 \mathrm{~ns}$ per $\mathrm{dB}$ gain was perfectly confirmed experimentally [6] and delays from $-8 \mathrm{~ns}$ in fast-light regime up to $32 \mathrm{~ns}$ in slow-light regime could be realized in these early demonstrations [6]. Some months later, higher effective gains could be achieved by inserting spectrally neutral attenuators between fibre segments to prevent an excessive amplification of the signal while fully preserving the delaying effect[8]. As shown in Fig. 1, delays could be extended up to $152 \mathrm{~ns}$ for a $40 \mathrm{~ns}$ input pulse, corresponding to a delay normalized to the pulse width of 3.6 [8]. Nevertheless, as theoretically predicted[7, 9, $10]$, the pulse experienced a very substantial broadening by a factor 2.4 for the maximal delay.

Even though impressive delays were obtained in these pioneering experiments they were achieved in kilometre-long fibres and these delays represent only a minor fraction of the total transit time. The corresponding group index change $\Delta n_{g}$ is in the $10^{-3}-10^{-2}$ range and the modification of the absolute group velocity is only marginal, far from the amazing results obtained in atomic media. Group index changes comparable to the natural group index in silica (1.46) are possible in a $\sim 4$ metre long fibre, but require a pump power of about $10 \mathrm{~W}$ to realize a sufficient gain. An experiment with 2-metre long fibre was carried out demonstrating the slowing of

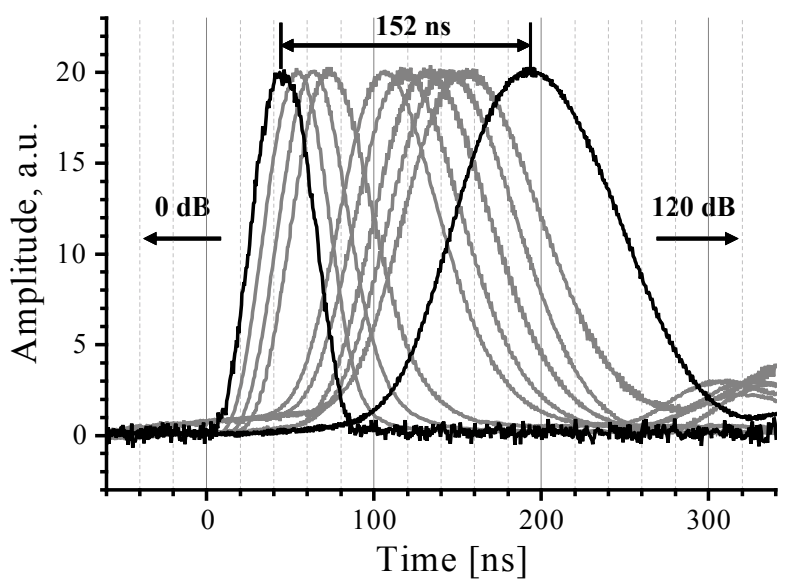

Fig. 1 Time waveforms of the probe pulses for different Brillouin gains, showing a time delay much exceeding the initial pulse width (42 ns) signal down to a velocity of $71,000 \mathrm{~km} \mathrm{~s}^{-1}$, corresponding to a group index of 4.26 or a speed reduction by nearly a factor of 3 using a pump power estimated to be $\sim 5 \mathrm{~W}[11]$.

Using the same experiment the EPFL team could also drastically accelerate the signal in a fast light configuration using the Brillouin loss process. They could reach the regime of superluminal propagation, namely a group velocity exceeding the vacuum light velocity. The maximal advancement attained $14.4 \mathrm{~ns}$, to be compared to the $10 \mathrm{~ns}$ of normal propagation time in the 2 metre fibre[11]. This is a situation of a negative group velocity and literally it means that the main feature of the signal exits the fibre before entering it. This can be actually visualized in Fig. 2 where it can be clearly seen that the peak of the pulse is simultaneously present at the input and the output 

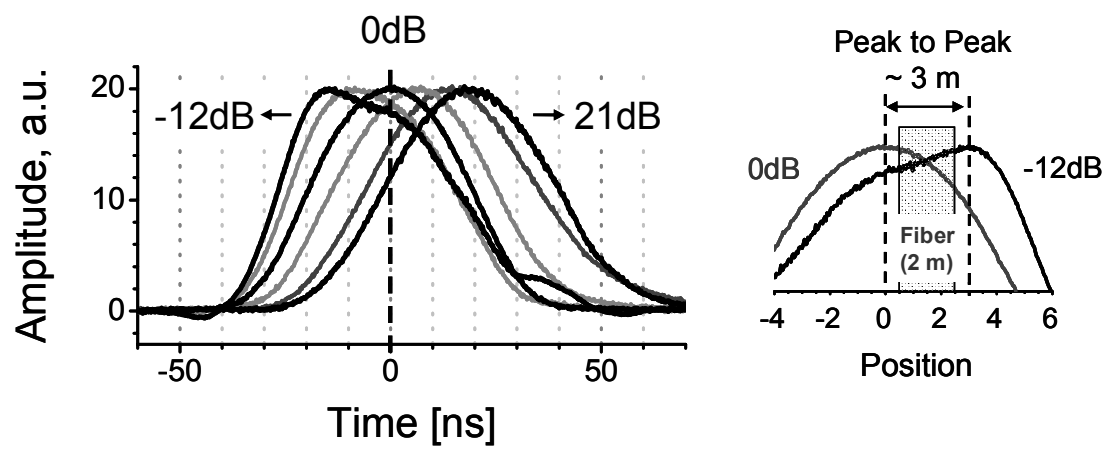

Fig. 2. Traces of the probe pulses for different Brillouin gains and losses in a 2-m test fiber, showing clear delay and advancement due to the modified group velocity. The inset compares the respective positions of the pulse propagating in normal conditions (OdB) and of the most advanced pulse (-12dB), demonstrating that in this case the pulse peak exits the fiber before entering it. This situation corresponds to a negative group velocity. The fiber length is represented by the shaded area.

of the fibre. These results were the first demonstration that a negative group velocity can actually be achieved in optical fibres.

However, despite these impressive achievements obtained using simple benchtop set-ups, it was soon identified that the narrowband nature of stimulated Brillouin scattering will make it unsuitable for broadband data communications, since the $30 \mathrm{MHz}$ linewidth of the gain spectral distribution practically restricts the full delaying effect only to pulses longer than $40 \mathrm{~ns}$. This stimulated the research towards different gain processes in optical fibres showing a larger intrinsic linewidth.

\section{ADVANCED SCHEMES USING SBS SPECTRAL SHAPING}

More recently stimulated Brillouin scattering (SBS) has proved to be an unprecedented and unmatched flexible tool for the generation of slow light regarding its spectral tailoring capability. Indeed, a large variety of gain spectral profile can be obtained by properly modulating the pump spectrum. When a monochromatic pump is used in the stimulated Brillouin interaction, the gain window appearing in the fiber transmission spectrum shows a Lorentzian shape whose characteristic spectral FWHM width is around $30 \mathrm{MHz}$ in conventional single-mode fibers pumped at $1.55 \mu \mathrm{m}$. However, when the pump is modulated the gain bandwidth is given by the convolution of the pump spectrum and the Brillouin gain curve. Hence the effective Brillouin gain spectrum $g(\Delta v)$ is given by:

$$
g(\Delta v)=P(\Delta v) \otimes g_{B}(\Delta v)
$$

where $\otimes$ denotes convolution, $P(\Delta v)$ is the normalized pump power spectral density (i.e. its integral is unity) and $g_{B}(\Delta v)$ is the natural Lorentzian gain of the Brillouin amplification process.

The effect of this convolution is shown in Fig. 3. By properly modulating the pump signal, it is possible to generate nearly any gain spectral distribution and to optimize the slow \& fast light effect in terms of bandwidth,
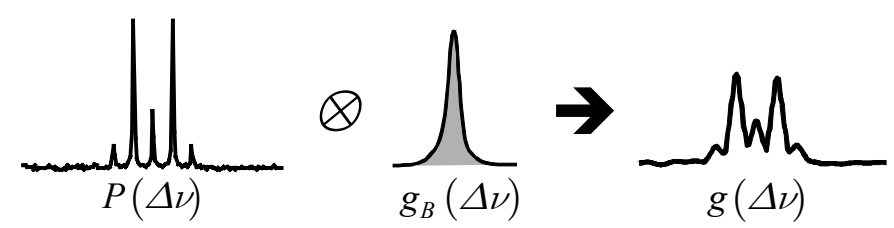

Fig. 3 The effective gain spectrum $g(\Delta v)$ is given by the convolution of the pump spectrum $P(\Delta v)$ with the natural gain distribution $g_{B}(\Delta v)$ of stimulated Brillouin scattering. This offers the possibility to synthesize nearly any gain spectral distribution. efficiency and distortion.

An essential step towards applications was made when it was demonstrated that the bandwidth of SBS-based slow light can be made arbitrarily large by actively broadening the pump spectrum using random direct current modulation of the pump laser [12]. A particularly useful case arises when the pump spectrum can also be approximated by a Lorentzian distribution. In such conditions, the effective Brillouin gain shape remains Lorentzian, but shows a width equal to the sum of the characteristic Brillouin gain width and the pump spectral width. Hence an adequate pump modulation can be used to broaden at will the Brillouin interaction, suppressing the inherent limitation related to the narrow natural linewidth.

Fig. 4 shows the delaying results of 2.7-ns pulses for several gain values. A linear dependence of the delay with the logarithmic gain is observed with a slope of approximately $0.092 \mathrm{~ns} / \mathrm{dB}$, as expected from the theory. By tuning the probe frequency appropriately, measurements in the Brillouin loss region were also performed, obtaining pulse advancement with the same linear dependence on the logarithmic gain. 


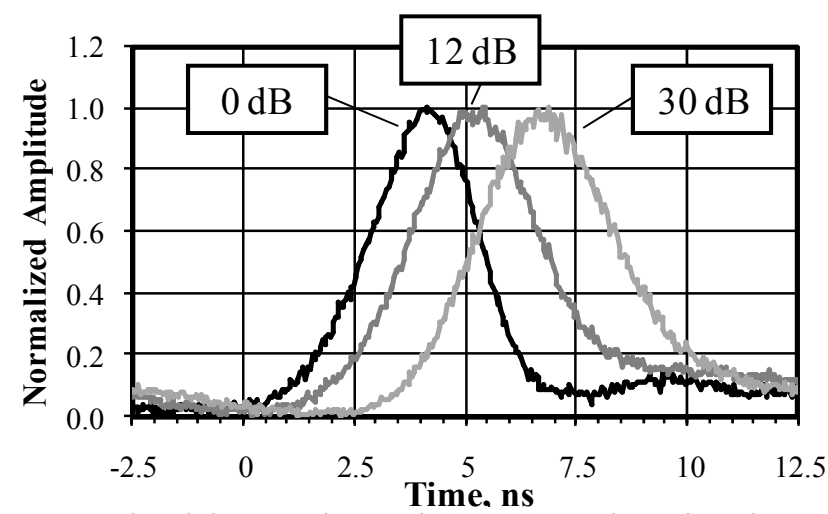

Fig. 4 Pulse delaying obtained using a 10 X broadened gain spectrum. The pulse width is $2.7 \mathrm{~ns}$.
This scheme was then replicated and improved successfully to recently ultimately reach a $10 \mathrm{GHz}$ bandwidth [13]. It was also demonstrated that the slowing efficiency can be substantially improved by departing from the Lorentzian shape to a more efficient finely tuned gain spectral distribution [14].

So far all these slow-light techniques suffer from the drawback of a significant amplitude change associated with the delaying effect. For instance a one-pulse width delaying gives rise to a large $30 \mathrm{~dB}$ pulse amplitude change using SBS. We have recently demonstrated that the high flexibility of SBS opens unprecedented achievements and offers the possibility to synthesize a gain spectral profile, so that signal delays or advances can be realized with an ideally absolute null amplitude change [15]. This can be obtained by the combination of gain and loss spectral profiles with identical depth but different width, resulting in a net zero gain and a differential delaying effect.

This is practically achieved by superposing in the frequency domain a SBS gain with linear gain $+G_{1}$ and a bandwidth $\Delta v_{1}$ on a SBS loss with negative linear gain (thus loss) $-G_{2}$ and a bandwidth $\Delta v_{2}$. They are generated using 2 distinct pump lasers placed at a frequency $\pm v_{B}$ above and below the signal frequency $v$, respectively. The bandwidth of each pump $\Delta v$ can be arbitrarily broadened and controlled using the scheme described above [12].

If the bandwidths of the gain and loss spectra are substantially different, e.g. $\Delta v_{2}$ » $\Delta v_{1}$, it is possible to obtain a significant time delay with nevertheless a zero linear gain. The effect is fully comparable to electromagnetically-induced transparency or coherent population oscillation, in which a transparency window is opened in the middle of an absorption line. Fig. 5 shows the obtained gain/loss spectral profile, demonstrating that a good compensation of gain and loss can actually be obtained. A situation identical to ideal electromagnetically-induced transparency (EIT) can be achieved, i.e. up to full transparency.

Delays and amplitudes were recorded for different pump levels in a $2 \mathrm{~km}$ fiber, AS SHOWN IN Fig. 6, and are comparable to the standard method [6], together with a maximum amplitude change of $\pm 1 \mathrm{~dB}$ that can be reasonably considered as a flat response. An equivalent delay using the non-compensated standard technique would result in a $12 \mathrm{~dB}$ amplitude change.

The gain compensation technique can also be applied to generate fast light, by simply swapping the frequency positions of Pump 1 and Pump 2. In this case the broadened pump generates a gain and the narrow pump burns a hole at the center of the gain spectrum. This configuration was also successfully experimentally tested and demonstrated [16].

The smart combination of gain and loss spectra using a multiple pump scheme was also used to extend the bandwidth well beyond $10 \mathrm{GHz}$ that was temporarily considered like the ultimate limit [17]. A $25 \mathrm{GHz}$ bandwidth was

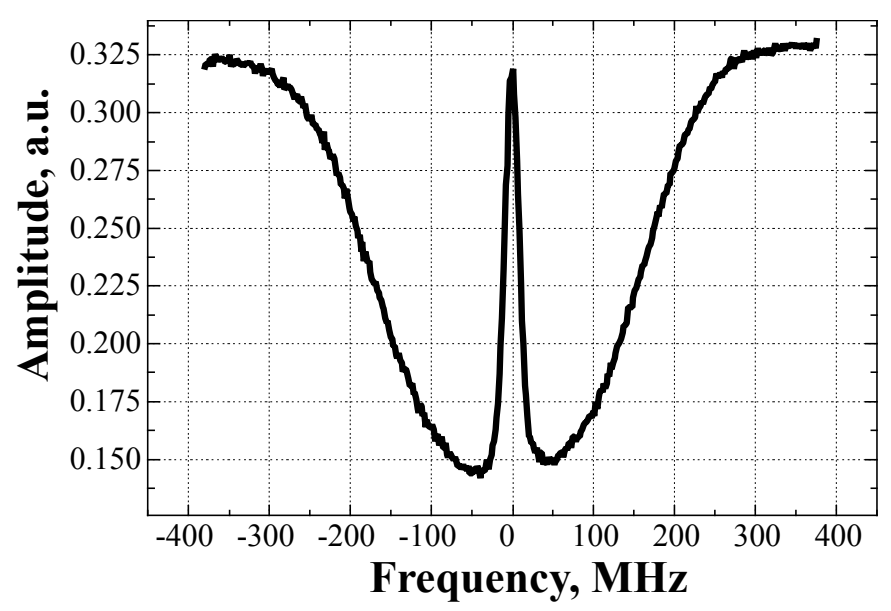

Fig. 5 Variation of the amplitude of the probe signal as a function of frequency after propagation through a $2 \mathrm{~km}$ fiber, showing a well-compensated SBS gain/loss profile.

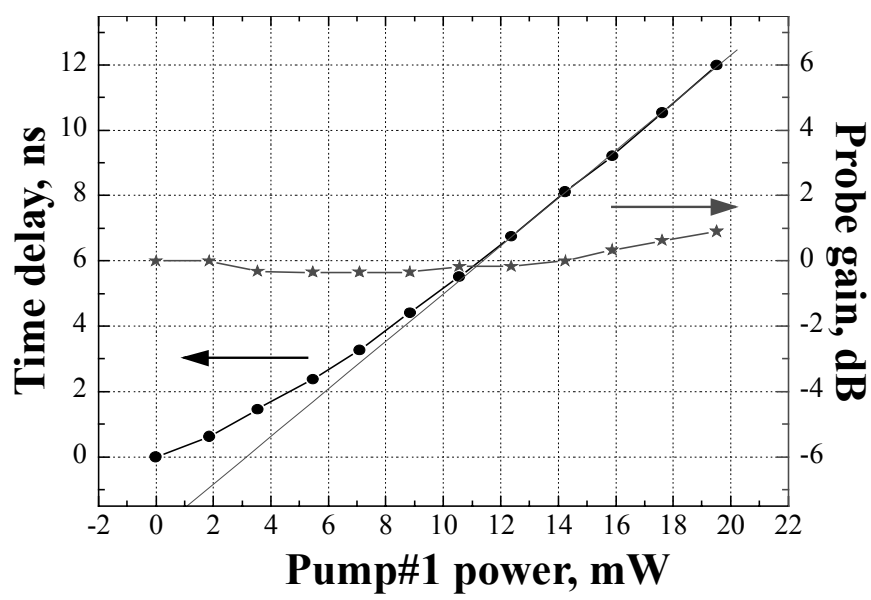

Fig.6. Delays and amplitudes as a function of Pump 1 power in a zero-gain slow light configuration. Power of Pump 2 is 12 times larger than power of Pump 1. 
experimentally demonstrated that can be potentially extended well beyond using additional pumps.

\section{CONCLUSIONS AND PERSPECTIVES}

Slow- and fast-light delay lines in optical fibres based on SBS shows unprecedented flexibility to realize innovative systems for the generation of optically controlled delays. SBS slow and fast light offers the outstanding property to make the shaping of the spectral resonance possible through the modulation of the pump. Attractive solutions were proposed in the recent years to overcome the challenges towards real applications. These solutions include the realization of broadband delays up to $25 \mathrm{GHz}$ and more, the optimization of the gain spectral distribution for effective delaying and slow distortion, the achievement of important delays in meterlong fibres and eventually the realization of delays with no amplitude change.

However, as pointed out by many valuable studies[18-21], the storage capability of slow- and fast-light delay lines based on spectral resonances, such as those presented in this review, are far below the most modest expectations for optically buffering a bit sequence. A simple analysis shows that storing a bit sequence longer than 5 bits remains unrealistic [9]. Moreover the performance required by modern telecommunication systems and optical digital processing in terms of delay and bandwidth would require a tremendous pumping power that turns the endeavour into a lost cause[20]. It means that slow- and fast-light delay lines are likely to miss the main target application that motivated the early research efforts, which is the all-optical buffering for the development of future all-optical routers[22]. For such an application, coherent optical storage looks more like a promising approach and recent results show that even SBS can realize this storage function in optical fibres[23]. This conclusion has pushed the research community to reconsider the potential applications and to examine the best niches to take advantage of the real slowing of light.

Keeping strictly the delaying function of slow light and considering the effect on a purely analog signal, it is already possible to realize systems that can produce tunable delays over slightly more than one signal period. This range of delays is definitely sufficient for applications in analog signal processing, such as the generation of true time delays in microwave photonics. This good match with the timing requirement for analog signals is further supported by the intrinsic linear response of SBS amplification, since linearity is another essential requirement for analog systems[24].

However, delaying is only one aspect of slow light and so far very few applications have taken advantage of the fact that light actually propagates slowly. Several studies have pointed out that a reduced velocity must have a significant impact on the light-matter interaction. For instance, it has been predicted that the efficiency of nonlinear optical effects must scale as the slowing factor squared[25-28]. In practice, it means that transformations and functions based on optical nonlinearities could be realized in much shorter distances, for instance, in $1 \mathrm{~cm}$ instead of $1 \mathrm{~m}$ if the light is slowed down to 1/10 of its normal group velocity. It has also been anticipated that the sensitivity of interferometers or sensing systems can be significantly improved by reducing the speed of the light signal [29-33]. However, no decisive experimental verifications have supported these predictions so far and certainly amazing and unexpected applications of this fascinating domain of slow light will be discovered in the coming years. This makes slow and fast light a yet opened field in which innovative and creative solutions are still to be found.

\section{ACKNOWLEDGEMENTS}

The author acknowledges the essential contributions of Kwang-Yong Song (currently at Chung-Ang University, Seoul, South Korea), of Miguel González-Herráez (currently at theUniversity of Alcalá-Madrid, Spain) and Sang-Hoon Chin (currently at EPFL, Switzerland).

\section{REFERENCES}

[1] L. V. Hau, S. E. Harris, Z. Dutton, and C. H. Behroozi, "Light speed reduction to 17 metres per second in an ultracold atomic gas," Nature, vol. 397, pp. 594-598, Feb 18, 1999.

[2] M. S. Bigelow, N. N. Lepeshkin, and R. W. Boyd, "Superluminal and slow light propagation in a roomtemperature solid," Science, vol. 301, pp. 200-202, Jul. 11, 2003.

[3] R. W. Boyd and D. J. Gauthier, "'Slow" and "fast" light," in Progress in Optics. vol. 43, 2002, pp. 497-530.

[4] R. W. Boyd, Nonlinear Optics. New York: Academic, 2003.

[5] J. T. Mok and B. J. Eggleton, "Photonics: Expect more delays," Nature, vol. 433, pp. 811-812, 2005.

[6] K. Y. Song, M. Gonzalez Herraez, and L. Thevenaz, "Observation of pulse delaying and advancement in optical fibers using stimulated Brillouin scattering," Optics Express, vol. 13, pp. 82-88, Jan 10, 2005.

[7] Y. Okawachi, M. S. Bigelow, J. E. Sharping, Z. M. Zhu, A. Schweinsberg, D. J. Gauthier, R. W. Boyd, and A. L. Gaeta, "Tunable all-optical delays via Brillouin slow light in an optical fiber," Physical Review Letters, vol. 94, Apr 22, 2005. 
[8] K. Y. Song, M. G. Herraez, and L. Thevenaz, "Long optically controlled delays in optical fibers," Optics Letters, vol. 30, pp. 1782-1784, Jul. 15, 2005.

[9] J. B. Khurgin, "Performance limits of delay lines based on optical amplifiers," Optics Letters, vol. 31, pp. 948-950, Apr. 1, 2006.

[10] Z. Zhu, D. J. Gauthier, Y. Okawachi, J. E. Sharping, A. L. Gaeta, R. W. Boyd, and A. E. Willner, "Numerical study of all-optical slow-light delays via stimulated Brillouin scattering in an optical fiber," J. Opt. Soc. Am. B, vol. 22, pp. 2378-2384, 2005.

[11] M. Gonzalez-Herraez, K. Y. Song, and L. Thevenaz, "Optically controlled slow and fast light in optical fibers using stimulated Brillouin scattering," Applied Physics Letters, vol. 87, Aug. 22, 2005.

[12] M. Gonzalez Herraez, K. Y. Song, and L. Thevenaz, "Arbitrary-bandwidth Brillouin slow light in optical fibers," Optics Express, vol. 14, pp. 1395-1400, Feb. 20, 2006.

[13] Z. M. Zhu, A. M. C. Dawes, D. J. Gauthier, L. Zhang, and A. E. Willner, "Broadband SBS slow light in an optical fiber," Journal of Lightwave Technology, vol. 25, pp. 201-206, Jan. 2007.

[14] A. Zadok, A. Eyal, and M. Tur, "Extended delay of broadband signals in stimulated Brillouin scattering slow light using synthesized pump chirp," Optics Express, vol. 14, pp. 8498-8505, Sep. 18, 2006.

[15] S. Chin, M. Gonzalez-Herraez, and L. Thevenaz, "Zero-gain slow \& fast light propagation in an optical fiber," Optics Express, vol. 14, pp. 10684-10692, Oct. 30, 2006.

[16] S. Chin, M. Gonzalez Herraez, and L. Thevenaz, "Zero-gain slow \& fast light propagation in an optical fiber," Optics Express, vol. 14, pp. 10684-10692, Oct. 30, 2006.

[17] K. Y. Song and K. Hotate, "25 GHz bandwidth Brillouin slow light in optical fibers," Optics Letters, vol. 32, pp. 217-219, Feb. 1, 2007.

[18] R. W. Boyd, D. J. Gauthier, A. L. Gaeta, and A. E. Willner, "Maximum time delay achievable on propagation through a slow-light medium," Physical Review A, vol. 71, Feb, 2005.

[19] R. S. Tucker, P. C. Ku, and C. J. Chang-Hasnain, "Slow-light optical buffers: Capabilities and fundamental limitations," Journal of Lightwave Technology, vol. 23, pp. 4046-4066, Dec. 2005.

[20] J. B. Khurgin, "Power dissipation in slow light devices: a comparative analysis," Optics Letters, vol. 32, pp. 163-165, Jan. 15, 2007.

[21] J. B. Khurgin, "Slow Light: Fundamentals \& Applications," in Optical Amplifiers and Their Applications/Coherent Optical Technologies and Applications: Optical Society of America, 2006, p. CThD3.

[22] R. W. Boyd, D. J. Gauthier, and A. L. Gaeta, "Applications of slow light in telecommunications," Optics \& Photonics News, vol. 17, pp. 18-23, Apr. 2006.

[23] Z. M. Zhu, D. J. Gauthier, and R. W. Boyd, "Stored light in an optical fiber via stimulated Brillouin scattering," Science, vol. 318, pp. 1748-1750, 2007.

[24] J. Capmany and D. Novak, "Microwave photonics combines two worlds," Nat Photon, vol. 1, pp. 319-330, 2007.

[25] M. Soljacic, S. G. Johnson, S. H. Fan, M. Ibanescu, E. Ippen, and J. D. Joannopoulos, "Photonic-crystal slow-light enhancement of nonlinear phase sensitivity," Journal of the Optical Society of America BOptical Physics, vol. 19, pp. 2052-2059, Sep. 2002.

[26] M. Soljacic and J. D. Joannopoulos, "Enhancement of nonlinear effects using photonic crystals," Nat Mater, vol. 3, pp. 211-219, 2004.

[27] A. B. Matsko, Y. V. Rostovtsev, H. Z. Cummins, and M. O. Scully, "Using Slow Light to Enhance Acousto-optical Effects: Application to Squeezed Light," Physical Review Letters, vol. 84, p. 5752, 2000.

[28] [M. M. Kash, V. A. Sautenkov, A. S. Zibrov, L. Hollberg, G. R. Welch, M. D. Lukin, Y. V. Rostovtsev, E. S. Fry, and M. O. Scully, "Ultraslow Group Velocity and Enhanced Nonlinear Optical Effects in a Coherently Driven Hot Atomic Gas," Physical Review Letters, vol. 82, p. 5229, 1999.

[29] Z. Shi, R. W. Boyd, R. M. Camacho, P. K. Vudyasetu, and J. C. Howell, "Slow-light Fourier Transform interferometer," Physical Review Letters, vol. 99, 2007.

[30] Z. M. Shi, R. W. Boyd, D. J. Gauthier, and C. C. Dudley, "Enhancing the spectral sensitivity of interferometers using slow-light media," Optics Letters, vol. 32, pp. 915-917, 2007.

[31] M. A. Terrel, M. J. F. Digonnet, and S. H. Fan, "Performance Limitation of a Coupled Resonant Optical Waveguide Gyroscope," Journal of Lightwave Technology, vol. 26, in press, 2008.

[32] M. J. F. Digonnet, "New technologies in fiber sensors," in Lasers and Electro-Optics, 2007 and the International Quantum Electronics Conference. CLEOE-IQEC 2007. European Conference on, 2007, pp. $1-1$.

[33] M. Gonzalez-Herraez, O. Esteban, F. B. Naranjo, and L. Thevenaz, "How to play with the spectral sensitivity of interferometers using slow light concepts and how to do it practically," in Third European Workshop on Optical Fibre Sensors, 1 ed. vol. 6619 Napoli, Italy: SPIE, 2007, pp. 661937-4. 